\title{
Article
}

\section{Giant planet migration during FU Orionis outbursts: 1D disc models}

\author{
Dunhill, Alex
}

Available at http://clok.uclan.ac.uk/21462/

Dunhill, Alex ORCID: 0000-0003-3951-0318 (2018) Giant planet migration during FU Orionis outbursts: 1D disc models. Monthly Notices Of The Royal Astronomical Society, 478 (3). pp. 3438-3446. ISSN 0035-8711

It is advisable to refer to the publisher's version if you intend to cite from the work. https://doi.org/10.1093/mnras/sty1235

For more information about UCLan's research in this area go to

http://www.uclan.ac.uk/researchgroups/ and search for <name of research Group>.

For information about Research generally at UCLan please go to http://www.uclan.ac.uk/research/

All outputs in CLoK are protected by Intellectual Property Rights law, including Copyright law. Copyright, IPR and Moral Rights for the works on this site are retained by the individual authors and/or other copyright owners. Terms and conditions for use of this material are defined in the policies page.

\section{CLoK}

Central Lancashire online Knowledge www.clok.uclan.ac.uk

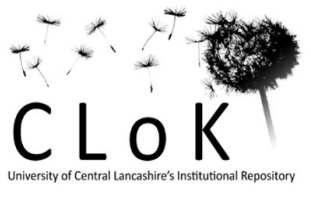




\title{
Giant planet migration during FU Orionis outbursts: 1D disc models
}

\author{
A. C. Dunhill` \\ Jeremiah Horrocks Institute for Mathematics Physics \& Astronomy, University of Central Lancashire, Preston PR1 2HE, UK
}

Accepted 2018 May 9. Received 2018 April 27; in original form 2016 August 24

\begin{abstract}
I present the results of semi-analytic calculations of migrating planets in young, outbursting circumstellar discs. Formed far out in the disc via gravitational fragmentation early on in its lifetime, these planets typically migrate at very slow rates and are therefore mostly expected to remain at large radii (such as is the case in HR 8799). I show that changes in the disc structure during FU Orionis outbursts affect the planet's ability to maintain a gap and can allow a massive giant planet's semimajor axis to reduce by almost 5 per cent in a single outburst under the most optimistic conditions. Given that a single disc will likely undergo $\sim 10$ such outbursts, this process can significantly alter the expected radial distribution for GI-formed planets.
\end{abstract}

Key words: accretion, accretion discs - planets and satellites: dynamical evolution and stability - planets and satellites: formation - planet-disc interactions - protoplanetary discs.

\section{INTRODUCTION}

Models of giant planet formation generally fall within two camps. The first group, known as core accretion models (CA; e.g. Pollack et al. 1996), builds solid materials for planetary cores up from initial dust grains that then undergo runaway gas accretion to become gas giants. The second group, gravitational instability models (GI; e.g. Boss 1998), proposes that young discs $(\lesssim 0.1 \mathrm{Myr})$ are massive and cool enough to fragment into bound clumps that then evolve to become giant planets and brown dwarfs, depending on the clump's initial mass and thermal evolution (e.g. Rice et al. 2015). There is a broad (but by no means universal) consensus that CA models are more realistic, but neither can be ruled out due to the lack of concrete differences between observational predictions from the different models. At present, the state-of-the-art here has been to attempt to diagnose the planet's internal structure - in general, CA models produce larger cores than GI models (e.g. Durisen et al. 2007). Given that we struggle to understand the internal structure of the Solar system planets such as Jupiter (e.g. Helled et al. 2014), this method is both ambiguous and difficult to apply.

A key observational constraint on the evolution of young circumstellar discs, such as those that would fragment and form planets under GI models, are FU Orionis (FUor) outbursts. These events are sudden increases (rise time $\sim 1 \mathrm{yr}$ ) by many orders of magnitude in luminosity, understood to be driven by an associated spike in accretion. From current statistics, it seems that these accretion events are sufficiently common that all young stellar objects should undergo approximately $5-10$ of these events during the lifetime of their discs (Hartmann 2009). It is therefore clear that if planets form via GI then they likely see their parent disc undergo multiple
FUor outbursts. Such massive luminosity (and therefore temperature) changes will undoubtedly alter the structure of the disc in which the GI-formed planets sit, altering their migration and affecting their eventual orbits once the disc disperses. If this process is able to alter the semimajor axis of a migrating giant by even 1 per cent during an outburst then neglecting it in population synthesis modelling of GI-formed planets will lead to incorrect predictions.

In this paper, I investigate the effect of an FUor outburst on a planet migrating through such a disc, focusing on the effect on the migration rate, using $1 \mathrm{D}$ viscous accretion disc models coupled to prescribed migration rates in a semi-analytic fashion. In Section 2 , I discuss the gap-opening criteria and its critical effect on the migration of a planet and argue that an FUor disc will force planets migrating in it to change migration regime. In Section 3, I describe the 1D numerical model used to calculate the structure of discs undergoing multiple FUor-type outbursts and follow the migration of embedded planets, the results of which I present in Section 4. I then discuss the limitations of these models, and the interpretation of the results in Section 5 before drawing conclusions in Section 6 .

\section{GAP OPENING}

Migrating planets are classified as undergoing either Type I or Type II migration ${ }^{1}$ depending primarily on their ability to open and maintain a gap in the surrounding disc. In the context of a standard, relatively low-mass protoplanetary disc, planets below approximately a Jupiter mass are unable to open a gap and migrate quite rapidly in the Type I regime, driven by resonant interactions with the disc (e.g. Tanaka, Takeuchi \& Ward 2002). More massive planets are able to isolate themselves from their disc by opening a gap and limiting 


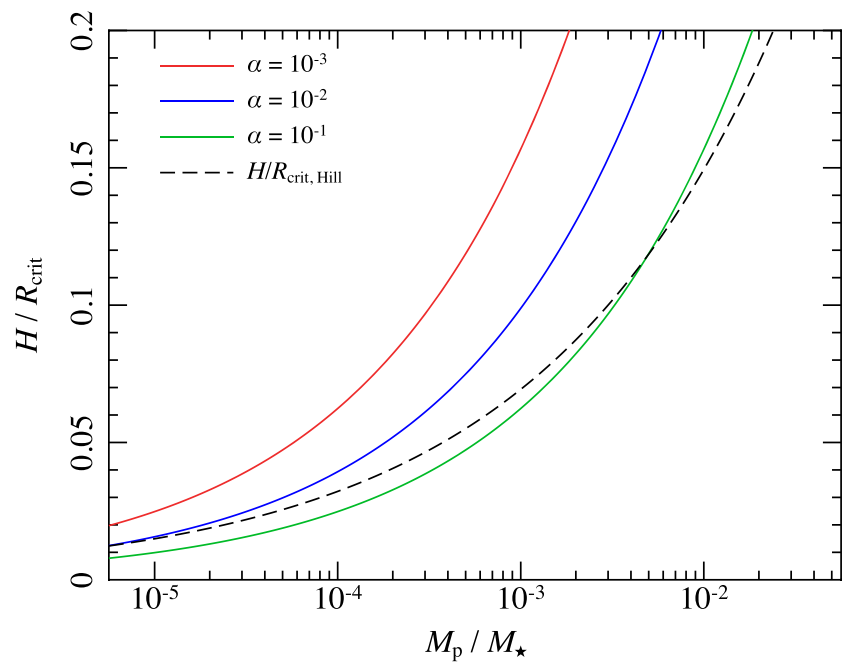

Figure 1. Values of the critical disc aspect ratio $H / R_{\text {crit }}$ for gap opening, for various values of the planet-star mass ratio $M_{\mathrm{p}} / M_{\star}$ and Shakura \& Sunyaev (1973) viscous parameter $\alpha$. Solid lines show values of $H / R_{\text {crit. visc }}$ (equation 1), and the dashed line is for $H / R_{\text {crit, }}$ Hill (equation 2). In order for the planet to maintain a gap in the disc, $H / R$ must be below both of these values.

the effect of these resonant torques, so their migration occurs at the slower Type II rates, where the planet's migration rate is limited by the viscous evolution of the disc.

The criteria for gap opening are not exact as they depend strongly on parameters of the disc, primarily its thickness, surface density, and viscosity. One method of determining if a planet will open a gap or not is to compare the time-scale on which a planet will clear co-orbital material (i.e. the gap- opening time-scale) with the timescale on which material will viscously spread back into the cleared gap (the gap-closing time-scale). Using the impulse approximation to estimate the former and assuming a gap width on the order of the scale height $H$ for the latter (e.g. Armitage 2010), one finds that the critical value of the disc aspect ratio $H / R$ is

$\frac{H}{R}_{\text {crit, visc }}=\left(\frac{8}{27 \pi} \frac{1}{\alpha}\right)^{1 / 5}\left(\frac{M_{\mathrm{p}}}{M_{\star}}\right)^{2 / 5}$.

However, viscous spreading is not the only consideration one must take into account for gap opening. The derivation of equation (1) assumes that the disc can be treated as being $2 \mathrm{D}$, which is only valid when the planet's Hill radius $R_{\text {Hill }}=a\left(M_{\mathrm{p}} / 3 M_{\star}\right)^{1 / 3}>H$. The classical understanding is that if this is violated then disc material will start to flow into the gap from regions more than $z=R_{\text {Hill }}$ above the mid-plane, which the planet is unable to prevent. This leads to a second critical value

$\frac{H}{R}_{\text {crit, Hill }}=\left(\frac{1}{3} \frac{M_{\mathrm{p}}}{M_{\star}}\right)^{1 / 3}$.

Fig. 1 compares the two values of $H / R_{\text {crit }}$ for different disc parameters. For a planet to maintain a gap, it is necessary that $H / R<\min \left(H / R_{\text {crit, visc }}, H / R_{\text {crit, Hill }}\right)$. From Fig. 1 , we can see that for representative values of $M_{\mathrm{p}} / M_{\star} \sim 10^{-3}$ and $\alpha \sim 10^{-2}$ the dominant (minimum) criterion is $H / R_{\text {crit, Hill, }}$ and the planet can only maintain a gap if $H / R \lesssim 0.1$.

However, simulations have shown that planets are able to maintain a gap even when this second criterion (equation 2) is violated (e.g. Duffell \& MacFadyen 2012, 2013; Zhu, Stone \& Rafikov 2013), questioning its usefulness. This can be understood by exam- ining the gap-opening process. The interaction between the planet and disc removes angular momentum from the planet, and this torque drives a spiral wave in the disc. It is the strength of this wave and how the disc reacts to it that determines whether or not a gap is opened (Rafikov 2002; Duffell \& MacFadyen 2012). In general, high-mass planets create a non-linear response that shocks close to the planet depositing the angular momentum there and driving disc material away from the planet's orbit, opening a gap. The relationship between the Hill radius and the disc thickness in this picture comes from the expectation that the wave will shock within the hill sphere, and the Mach number $\mathcal{M}=H / R^{-1}$ of the shock. Conversely, a weaker shock with a lower Mach number may deposit the angular momentum outside its Hill radius and still open a gap, violating equation (2). It may therefore be considered a sufficient, but not necessary, criterion for gap opening. While this still does not combat against matter flowing into the gap from outside the mid-plane, it does provide a mechanism for opening (an albeit shallower) gap at lower planet masses.

In a standard protoplanetary disc, these criteria are usually fulfilled by a giant planet in the Jupiter mass regime, allowing it to maintain a gap and migrate at Type II rates. During an FUor-type outburst however, the outer regions of the disc will become much hotter due to the increased luminosity of the inner $\sim 0.5$ au disc (e.g. Zhu et al. 2008). This increased temperature will cause the outer disc to thicken significantly and, if it becomes thicker than the planet's Hill radius, overwhelm the planet and close the gap if we take equation (2) to be a necessary criterion for gap opening. Therefore, although FUor events are likely to be short-lived ( $\tau_{\mathrm{FU} o r} \sim 100 \mathrm{yr}$; Hartmann 2009 ), a planet that forms early enough that it sees such an event will have its orbital evolution significantly affected. To assess the effect of gaps opened even in violation of the Hill criteria, I will consider models where (i) both criteria are considered and (ii) only the viscous criterion is considered to be necessary for gap opening.

\section{NUMERICAL MODEL}

The basis of the model used is the traditional 1D viscous accretion disc model (e.g. Pringle 1981) where the surface density $\Sigma$ is evolved according to

$$
\frac{\partial \Sigma}{\partial t}=\frac{1}{R} \frac{\partial}{\partial R}\left[3 R^{1 / 2} \frac{\partial}{\partial R}\left(v \Sigma R^{1 / 2}\right)\right],
$$

where $R$ is stellocentric radius and $v$ is the shear viscosity, parametrized here using the Shakura \& Sunyaev $\alpha$ parametrization:

$v(R)=\alpha H^{2} \Omega$.

$\Omega$ is the orbital frequency of material in the disc and $H$ is the disc thickness.

\subsection{Energy equation and outburst model}

In Section 2, I showed that the disc thickness $H$ is the key to whether or not a migrating planet is able to open a gap in its disc, which in turn dictates its migration regime (and therefore its migration rate). In order to self-consistently calculate $H$, it is necessary to include both an energy equation that is evolved alongside equation (3) and a model for the outburst itself. To this end, I use the energy equation from Cannizzo (1993), see also Wang (2015), combined with a toy outburst model based on that of Stamatellos, Whitworth \& Hubber 
(2011). The disc mid-plane temperature $T_{\mathrm{m}}$ is evolved according to

$$
\begin{aligned}
\frac{\partial T_{\mathrm{m}}}{\partial t}= & \frac{Q_{+}-Q_{-}}{c_{\mathrm{p}} \Sigma}+\frac{3 v}{R} \frac{\partial}{\partial R}\left(R \frac{\partial T_{\mathrm{m}}}{\partial R}\right) \\
& -\frac{\mathcal{R} T_{\mathrm{m}}}{\mu c_{\mathrm{p}}} \frac{1}{R} \frac{\partial\left(R u_{\mathrm{R}}\right)}{\partial R}-u_{\mathrm{R}} \frac{\partial T_{\mathrm{m}}}{\partial R},
\end{aligned}
$$

where $Q_{+}$and $Q_{-}$are, respectively, the heating and cooling rates. $c_{\mathrm{p}}$ is the specific heat capacity with constant pressure, for which I adopt the functional form of $c_{\mathrm{p}}\left(T_{\mathrm{mid}}\right)$ given by Cannizzo (1993) and

$u_{\mathrm{R}}=-\frac{3}{\Sigma R^{1 / 2}} \frac{\partial}{\partial R}\left(v \Sigma R^{1 / 2}\right)$

is the vertically averaged radial velocity in the disc. The second term on the RHS of equation (5) is the viscous transport of energy radially through the disc (compare the form of this term with the form of equation (1) itself; Mineshige 1986; Cannizzo 1993). The third term represents compressional heating (i.e. $P \mathrm{~d} V$ work) while the fourth term is the contribution from advective transport.

In order to close the set of equations, I calculate at each timestep two additional temperatures, $T_{\mathrm{ir}}(R)$ and $T_{\mathrm{S}}(R)$. The former represents the effect of stellar irradiation through the disc. I take the form derived by Ruden \& Pollack (1991), which is valid for $R \gg R_{\star}$ where $R_{\star}$ is the stellar radius:

$T_{\mathrm{ir}}^{4}=T_{\star}^{4}\left[\frac{2}{3 \pi}\left(\frac{R_{\star}}{R}\right)^{3}+\frac{1}{2}\left(\frac{R_{\star}}{R}\right)^{2}\left(\frac{H}{R}\right)\left(\frac{\mathrm{d} \ln H}{\mathrm{~d} \ln R}-1\right)\right]$.

To avoid numerical problems, I follow Hueso \& Guillot (2005) and take $\mathrm{d} \ln H / \mathrm{d} \ln R=9 / 7$. This is consistent with a flaring disc and is only significant at large $R$. I have compared runs with $\mathrm{d} \ln H / \mathrm{d} \ln R$ calculated self-consistently within the code and it does not make a significant difference to the evolution of $T_{\text {mid }}$ or $\Sigma$ inside $\sim 200 \mathrm{au}$, but it avoids considerable numerical instability.

The second additional temperature I calculate is the effective disc surface temperature, $T_{\mathrm{s}}(\mathrm{R})$. I use the method of Wang (2015) where constant vertical heat flux through the disc is assumed. This gives

$T_{\mathrm{s}}^{4}=\frac{8}{8+3 \kappa \Sigma}\left(T_{\mathrm{m}}^{4}+\frac{3 \kappa \Sigma}{8} T_{\mathrm{ir}}^{4}+\frac{3 \kappa \Sigma}{8} T_{\mathrm{am}}^{4}\right)$,

where $T_{\mathrm{am}}=5 \mathrm{~K}$ is the temperature of the ambient medium surrounding the disc and acts as a floor preventing further numerical problems. I adopt the Bell \& Lin (1994) method for calculating opacities $^{2} \kappa\left(\rho_{\text {mid }}, T_{\mathrm{m}}\right)$, where I assume that the mid-plane gas (volume) density $\rho_{\mathrm{m}}$ is the vertically averaged value $\rho_{\mathrm{m}}=\Sigma / 2 H$.

The local heating and cooling rates in equation (5) can now be calculated using these quantities. First, the heating rate $Q_{+}$is

$Q_{+}=\frac{9}{4} \Sigma \nu \Omega^{2}+2 \sigma_{\mathrm{SB}} T_{\mathrm{ir}}^{4}+2 \sigma_{\mathrm{SB}} T_{\mathrm{am}}^{4}$,

where $\sigma_{\mathrm{SB}}$ is the Stefan-Boltzmann constant. Here, the first term on the RHS is the usual viscous heating in the disc and the second and third terms represent heating from irradiation from the central star and ambient material, respectively.

Local cooling in the disc is given by

$Q_{-}=2 \sigma_{\mathrm{SB}} T_{\mathrm{sur}}^{4}$,

${ }^{2}$ I ran comparative models using both the modification to the Bell \& Lin (1994) opacities proposed by Bell et al. (1997) and the separate opacity tables given by Zhu et al. (2007) and found negligible change in the overall results which is simply the blackbody emission due to the hot disc surface.

From this temperature $T_{\mathrm{m}}$, I am able to calculate a disc scale height $H$. However, the discs I consider are massive and may therefore be unstable to self-gravity, which can strongly alter the vertical structure (and thus the thickness) of a disc. For this reason, I adopt the solution found by Bertin \& Lodato (1999):

$H=\frac{c_{\mathrm{s}}^{2}}{4 G \Sigma} \frac{1}{Q_{\mathrm{T}}^{2}}\left[\sqrt{1+\frac{8}{\pi} Q_{\mathrm{T}}^{2}}-1\right]$,

where $Q_{\mathrm{T}}$ is the Toomre (1964) parameter

$Q_{\mathrm{T}} \simeq \frac{c_{\mathrm{S}} \Omega}{\pi G \Sigma}$

This form of $H$ neatly captures the two limits for non-self-gravitating discs with thickness $H_{\text {nsg }}$ and self-gravitating discs with $H_{\mathrm{sg}}$, respectively, given by (e.g. Lodato 2007; Kratter \& Lodato 2016)

$H_{\mathrm{nsg}}=\frac{c_{\mathrm{s}}}{\Omega}$

and

$H_{\mathrm{sg}}=\frac{c_{\mathrm{s}}^{2}}{\pi G \Sigma}$.

I adopt a toy model for the outburst itself, a much simplified version of the model developed by Stamatellos et al. (2011), which in turn was based on the results of detailed disc modelling by Zhu, Hartmann \& Gammie (2009a), Zhu et al. (2009b, 2010a), and Zhu, Hartmann \& Gammie (2010b). I ran each simulation for a time of $10^{4} \mathrm{yr}$, with outbursts triggered at $t_{\text {burst }}=2.5 \times 10^{3}, 5 \times 10^{3}$, and $7.5 \times 10^{3} \mathrm{yr}$ and each outburst lasting for $\Delta t_{\text {burst }}=200 \mathrm{yr}$. When not in outburst the protostar has a luminosity of $L_{\star}=1.14 \mathrm{~L}_{\odot}$, which is the luminosity for an accretion rate of $\dot{M}_{\star}=10^{-7} \mathrm{M}_{\odot} \mathrm{yr}^{-1}$ on to an $M_{\star}=0.8 \mathrm{M}_{\odot}$. During an outburst the star's luminosity jumps to $L_{\star}=L_{\text {burst }}$ for which I use values of $L_{\text {burst }}=150,200$, and $250 \mathrm{~L}_{\odot}$ only altered between runs. The protostellar luminosity is used to tie the outburst model into the $1 \mathrm{D}$ disc model via the stellar temperature $T_{\star}$ in equation (7). This is calculated from the luminosity using the Stefan-Boltzmann law, i.e. $T_{\star}^{4}=L_{\star} / 4 \pi \sigma_{\mathrm{SB}} R_{\star}^{2}$, where I take $R_{\star}=3 \mathrm{R} \odot$.

Operationally, I use a grid equispaced in $R^{1 / 2}$ between $R=0.5$ and $R=10^{4}$ au containing 19860 cells. Advective quantities (i.e. $u_{R}$ ) are calculated using a first-order upwinding method. For all runs, I use $M_{\star}=0.8 \mathrm{M}_{\odot}$ and disc mass $M_{\mathrm{d}}=0.2 \mathrm{M}_{\odot}$. The initial surface density is given by

$\Sigma(R)=\frac{M_{\mathrm{d}}}{2 \pi R_{0} R} \exp \left(-R / R_{0}\right)$,

where $R_{0}=100$ au is a scaling radius. The initial temperature of the disc is set so that $T_{\mathrm{m}}(R)=T_{\mathrm{ir}}(R)$.

In addition to $L_{\text {burst }}$ and $M_{\mathrm{p}}$, I vary $\alpha$ in equation (4), using $\alpha=10^{-1}, 10^{-2}$, and $10^{-3}$. The evolution of the disc model's aspect ratio $H / R$ is shown in Fig. 2 for the case of the run with $\alpha=10^{-2}$ and $L_{\text {burst }}=200 \mathrm{~L}_{\odot}$ before, during, and after the initial outburst at $t=2500 \mathrm{yr}$.

\subsection{Planet migration rates}

In this work, I do not calculate torques on the planet self-consistently (as done by e.g. Alexander \& Armitage 2009), but instead adopt a semi-analytic approach. I do this as test runs show that the torque formalism of Lin \& Papaloizou (1986) used in 1D codes does not produce the correct behaviour when the planet is no longer in the Type II regime. As the aim of this work is to test how giant planets 


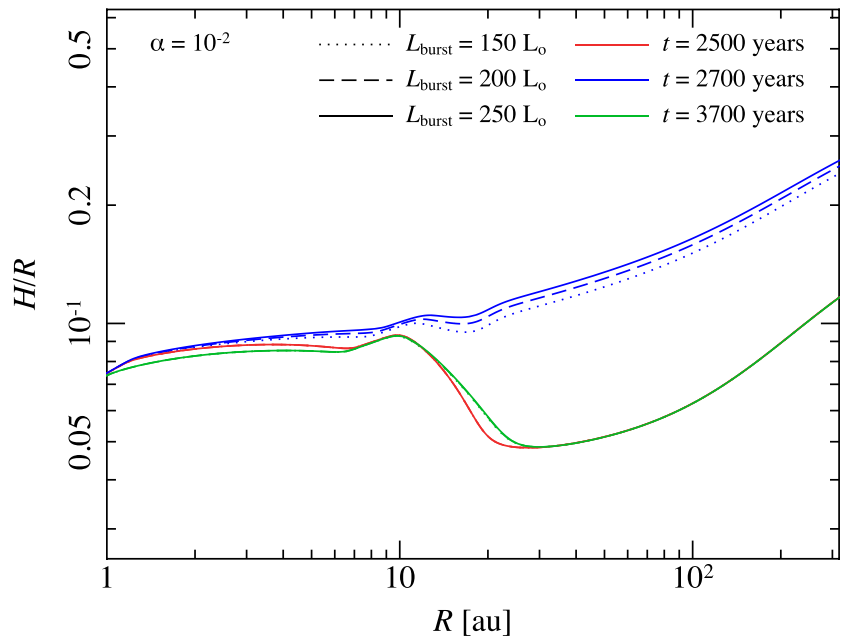

Figure 2. Evolution of the disc aspect ratio $H / R$ as a function of radius before (red lines), during (blue), and after (green) the initial outburst at $t$ $=2500 \mathrm{yr}$, for different burst luminosities $L_{\text {burst }}=150$ (dotted lines), 200 (dashed), and 250 (full) $\mathrm{L}_{\odot}$, for discs with $\alpha=10^{-2}$. The outburst primarily affects the disc at radii $R \gtrsim 10$ au, as the regions within this are already quite hot $\left(T \sim 10^{2}-10^{3} \mathrm{~K}\right)$ and optically thick. Outside the burst, the lines for different burst luminosities are indistinguishable.

behave once their disc is thick enough for them to leave the Type II regime, this is less than satisfactory.

I start with a planet placed at $R=a_{\mathrm{p}}=100$ au from the star, and at each step calculate the aspect ratio $H / R$ for the cell in which the planet resides using equation (11). I then use the criteria described in Section 2 to determine if the planet is able to open a gap, and therefore which migration regime it is in. I then move the planet radially inward using the migration rates given by Tanaka et al. (2002) for Type I

$$
\left(\frac{\mathrm{d} a_{\mathrm{p}}}{\mathrm{d} t}\right)_{\mathrm{I}}=2(1.364+0.541 q) \frac{M_{\mathrm{p}}}{M_{\star}}\left(\frac{H}{R}\right)^{-2} \frac{a_{\mathrm{p}}^{2} \Sigma}{M_{\star}} a_{\mathrm{p}} \Omega
$$

(where $q$ is the surface density power-law exponent, such that $\Sigma \propto$ $R^{-q}$ ) and Bate et al. (2003) for Type II

$$
\left(\frac{\mathrm{d} a_{\mathrm{p}}}{\mathrm{d} t}\right)_{\mathrm{II}}=\frac{3 \alpha}{2}\left(\frac{H}{R}\right)^{2} a_{\mathrm{p}} \Omega .
$$

It is interesting to note that the different migration rates scale with $H / R$ in opposite ways, with $\mathrm{d} a_{\mathrm{p}} / \mathrm{d} t \propto(H / R)^{-2}$ for Type I planets and $\mathrm{d} a_{\mathrm{p}} / \mathrm{d} t \propto(H / R)^{2}$ for Type II. Also noteworthy is the fact that only Type I rates are sensitive to the planet mass, and only Type II rates are sensitive to the viscosity parameter $\alpha$.

\section{RESULTS}

\subsection{Both gap-opening criteria}

It is clear from Fig. 1 that for the majority of the planet masses and disc viscosities considered here the dominant criteria for gap opening are the Hill criteria (equation 2), and that higher mass planets are better able to maintain a gap - meaning that lower mass planets are more likely to fall into the Type I migration regime during outburst. However, from equation (16) we see that the Type I migration rate is sensitive to the planet mass, meaning that only for higher mass planets will this change of migration regime makes a significant difference to $a_{\mathrm{p}}$. As Type I rates are insensitive to $\alpha$, its

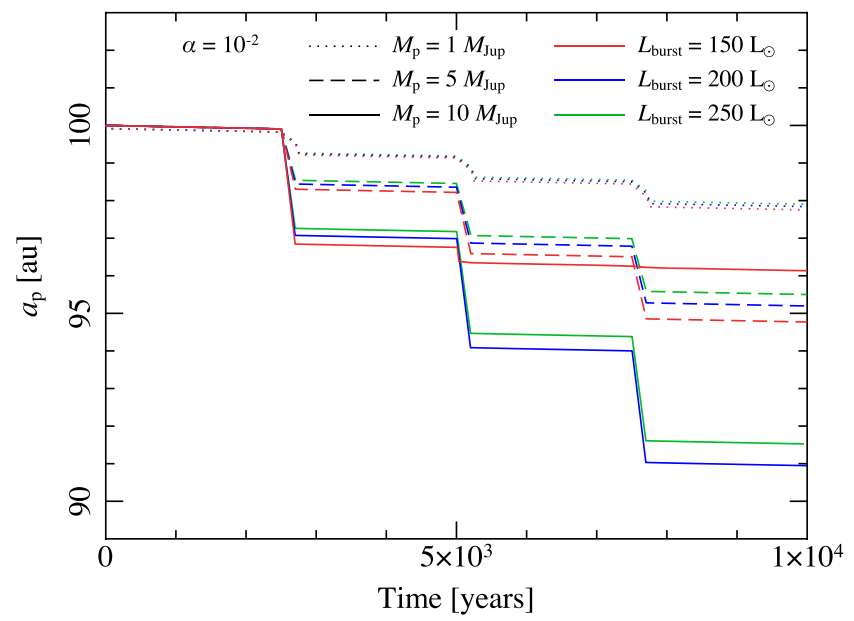

Figure 3. Semimajor axis as a function of time for all models with $\alpha=10^{-2}$ that used both gap-opening criteria. Dotted, dashed, and solid lines are for planet masses $M_{\mathrm{p}}=1,5$, and $10 M_{\mathrm{Jup}}$, respectively, while red, blue, and green lines indicate outburst luminosities $L_{\text {burst }}=150,200$, and $250 \mathrm{~L}_{\odot}$. When not in outburst the planets migrate at slow Type II rates (equation 16), and in nearly all cases at much faster Type I rates during outburst (equation 17). For the lowest outburst luminosity and highest planet mass run (solid red line), the disc only becomes thick enough for this to occur during the first outburst.

only major effect in these models is in setting the Type II migration rates when not in outburst and in its contribution to the internal disc heating in equation (5).

In Fig. 3, I plot $a_{\mathrm{p}}$ as a function of time for all runs with $\alpha=10^{-2}$. In all but one of these runs the results are qualitatively the same and quantitatively very similar. When not in outburst, the planets migrate at slow Type II rates (which on the time-scale considered here contributes negligibly to the change in $a_{\mathrm{p}}$ for $\alpha=10^{-2}$ ). During the outbursts, migration switches to Type I as expected and the planets move in much more rapidly until the outburst ends, at which point the planet returns to Type II migration. The minor differences in migration rates between runs with the same planet mass and viscosity parameter but with different outburst luminosity stem from the Type I migration rate's inverse dependence on $H / R$ (equation 16). As long as the outburst is sufficiently bright to heat the disc until it is thick enough that the planet's gap will close, then runs with lower outburst luminosities actually allow the planet to migrate faster.

The only runs that break the pattern above are those with low outburst luminosities and high planet masses $\left(L_{\text {burst }}=150 \mathrm{~L} \odot\right.$, $\left.M_{\mathrm{p}}=10 M_{\mathrm{Jup}}\right)$. For all values of $\alpha$, these runs behave as the others during the first outburst. However, for the second and third outbursts, the disc has evolved such that the outburst luminosity is not high enough to cause gap closing by disc thickening, so the planet continues to migrate at Type II rates even during outbursts.

\subsection{Viscous gap-opening criterion only}

As noted in Section 2, it has been shown that the Hill criterion for gap opening is not always a necessary one. To test the effect of planets that are able to open gaps without satisfying equation (2), I have run an equivalent series of calculations that use only the viscous gap-opening condition (equation 1). From Fig. 1, we see that now using only this critical value of $H / R$ to determine gap opening it is easier for a lower mass planet to maintain a gap during 


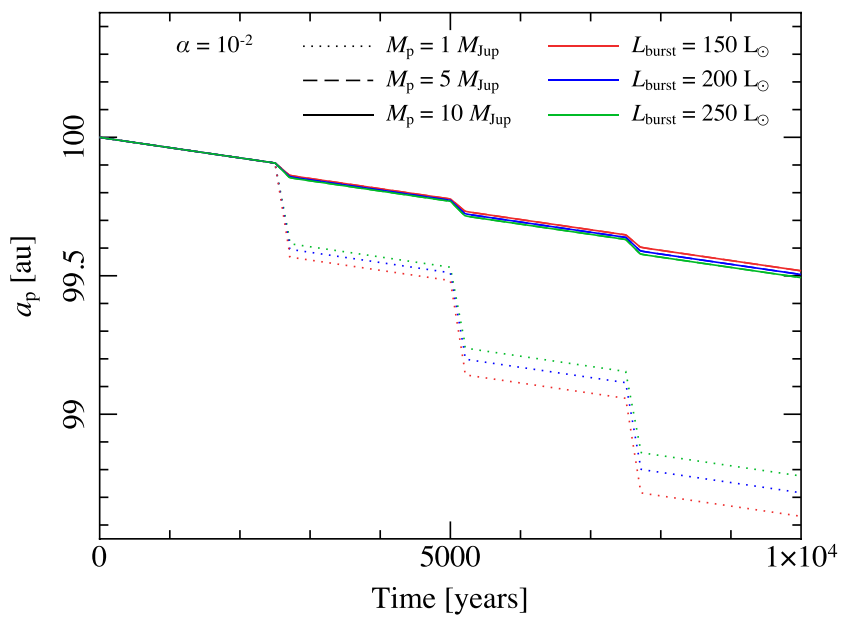

Figure 4. As Fig. 3 but for models where only the viscous gap-opening criterion (equation 1) is used. In this case, only the lowest mass planets $\left(M_{\mathrm{p}}=M_{\mathrm{Jup}}\right)$ actually enter the Type I migration regime during the outbursts, and even then, the change in semimajor axis is modest. The slight increase in migration rate for the higher mass planets is due to the $\mathrm{d} a_{\mathrm{p}} / \mathrm{d} t \propto(H / R)^{2}$ scaling for Type II migration (equation 17). Note that the $y$-axis scale is different from Fig. 3.

an outburst, as $H / R_{\text {crit }}$ is higher for a given value of $\alpha$. This is indeed borne out in the numerical models, the results of which are shown in Fig. 4 for the case of $\alpha=10^{-2}$.

Indeed, in all of the runs with modest viscosity $\left(\alpha<10^{-1}\right)$ the only planets that are not able to maintain a gap in the disc during outburst are the Jupiter mass ones. For models with $\alpha=10^{-1}$, the $M_{\mathrm{p}}=1$ and $5 M_{\text {Jup }}$ planets enter the Type I migration regime during the outbursts, along with the $10 M_{\text {Jup }}$ run with $L_{\text {burst }}=250 \mathrm{~L}_{\odot}$ model. However, in these cases the increase in migration rate is even more modest than in the runs using both gap-opening criteria. This is due to the increase in disc thickness required to close the gap on the one hand and the $\mathrm{d} a_{\mathrm{p}} / \mathrm{d} t \propto(H / R)^{-2}$ for Type I migration given in equation (16).

These models likely represent an extreme case for gap opening and closing. Even taking it as true that a planet of arbitrarily low mass could open a gap by depositing angular momentum into surrounding disc material, in some cases the discs in the calculations presented here become so thick that the scale height is greater than the disc's Hill radius by as much as a factor of three. In these cases, in a real 3D disc one would expect disc material to simply flow into the gap from outside the mid-plane and cause the gap to close. While in this case the 'thin disc' approximation used here is well and truly a bad one, it underlines the need for full 3D simulations to fully capture all the important effects (see Appendix A for some simplified 3D simulations of how gap opening and closing proceeds in the situation described here).

\section{DISCUSSION}

\subsection{Interpretation}

The results of these 1D calculations in Section 4.1 show that it is indeed possible for gap closing by a heated disc during a FUor-type outburst to significantly alter the semimajor axis of a migrating planet, potentially allowing planets formed at large radii by GI to move inward more rapidly than traditional models would predict. In Fig. 5, I plot estimates for the maximum possible change in

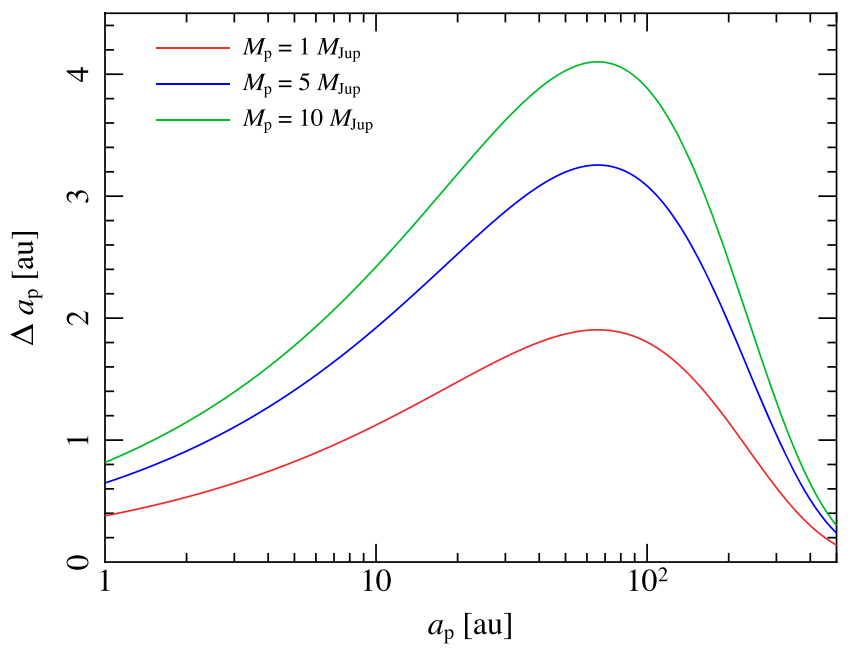

Figure 5. Maximum possible change in semimajor axis $\Delta a_{\mathrm{p}}$ during a single outburst against semimajor axis $a_{\mathrm{p}}$ for different planet masses $M_{\mathrm{p}}$. Calculated using equation (16) and assuming that the aspect ratio is the minimum possible while still being large enough to force gap closing (i.e. $\left.H / R=H / R_{\text {crit }}\right)$ and that the disc surface density $\Sigma$ is given by equation (15). These values are for protostellar mass $M_{\star}=0.8 \mathrm{M}_{\odot}$ and disc mass $M_{\mathrm{d}}=0.2 \mathrm{M}_{\odot}$.

semimajor axis, $\Delta a_{\mathrm{p}}$ during an individual such outburst as a function of the semimajor axis $a_{\mathrm{p}}$ and for different values of the planet mass $M_{\mathrm{p}}$. This is calculated by taking equation (16) and assuming that $H / R=H / R_{\text {crit, Hill }}$ and that $\Sigma$ is as given in equation (15).

It is worth noting that these are the maximum possible values for a system with a protostellar mass of $M_{\star}=0.8 \mathrm{M}_{\odot}$ and a disc mass $M_{\mathrm{d}}=0.2 \mathrm{M}_{\odot}-$ by their very nature the star-disc mass ratio will evolve with time. If we consider planets formed even earlier when the disc is more massive (and the protostar less massive) then the change in semimajor axis in a single outburst can be far greater. $\Delta a_{\mathrm{p}}$ scales linearly with $\Sigma$ (and therefore linearly with $M_{\mathrm{d}}$ ) and scales with $M_{\star}^{-5 / 6}$. Therefore, at earlier times a system with the same total mass but with a disc twice as massive $\left(M_{\mathrm{d}}=0.4 \mathrm{M}_{\odot}\right.$ and $\left.M_{\star}=0.6 \mathrm{M}_{\odot}\right)$ increases $\Delta a_{\mathrm{p}}$ by a factor a little over 3.5 times those shown in Fig. 3. This allows planets formed at even earlier times to migrate very rapidly indeed under the influence of an outburst.

The current consensus is that all young protostars undergo multiple (5-10) outbursts during the lifetime of their discs (Hartmann 2009). Using Fig. 5 as a basis, planets formed during this period at $R \sim 100$ au distances from the star will be able to lose on the order many tens of percent of their initial semimajor axis over the course of the outburst phase. The exact value requires more exact modelling taking into account not only the evolution of the disc surface density and temperature, as I consider here, but also including hydrodynamical effects. Indeed, using more recent parametrizations of the Type I migration rate provided by Paardekooper et al. (2010) for these estimates proves disastrous $-\Delta a_{\mathrm{p}}$ drops to less than $10^{-3}$ au for a single outburst for all planet masses. Paardekooper et al. (2010) take into account the adiabatic horseshoe drag that is likely to be dominant in optically thick discs, unlike the isothermal rates of Tanaka et al. (2002), but the calculations were made for 2D discs whereas the latter considers discs in 3D. Indeed, it is likely that neither rate is accurate in the regime considered here as it deals with extremely thick, hot discs, and very massive planets that are not usually thought of in the Type I migration framework. 


\subsection{Limitations and omissions}

There are many aspects of the physical systems observed that my simple models do not consider. I make no attempt to directly model the outburst mechanism itself, instead taking a toy version of the models developed by Zhu et al. (2009a,b, 2010a,b) and Stamatellos et al. (2011). As the region of the disc that is important for my models is well outside the $\sim 1$ au region where the outburst is generated this is unlikely to affect the result except in so far as the outburst parameters in my models differ from those generated in self-consistent models (e.g. my outbursts have a constant luminosity that is neither consistent with observations nor physical models) and are of fixed duration.

Recent near-infrared observations of FUors show strongly nonaxisymmetric features (Liu et al. 2016), which my 1D treatment is fundamentally incapable of capturing. However, the scale of these features is large compared to the 100 au scale I am interested in (typically many hundreds of au in size in the observations of Liu et al. 2016). While the smallest of these may to some extent affect the evolution of the disc near where my planets start, it is unlikely to dominate.

Although I attempt to account for the effects of GI in my treatment by calculating the disc scale height $H$ using a function (equation 11) that switches between the non-self-gravitating and self-gravitating limits as appropriate such an approach only gets one so far, especially given that I do not treat the interaction between the planet and disc self-consistently. This may be important at the edge of the planet's gap outside of outbursts where one expects a pressure bump and therefore an increase in the scale height (Rice et al. 2006). I further do not consider the increased viscosity generated by GI-driven spirals (e.g. Lodato 2008; Forgan et al. 2011; Kratter \& Lodato 2016) due to numerical instabilities this created in the code. The likely outcome of this is that the disc viscosity would be higher when not in outburst and lower when in outburst due to the heated disc becoming stable to its own self-gravity. However, given the long dynamical time at the distances considered here compared with the short duration of the outburst it is unclear what the full effect might be, as I find that the disc quickly cools back to its equilibrium conditions once the outburst is over, and there may not be enough time for the increased stability to manifest.

Indeed, the gap-opening criteria I use (equations 1 and 2) are at best not accurate for the parameters considered here, and the usefulness of the Hill criterion in particular is an issue of contention (see the discussion in Section 2). Malik et al. (2015) showed using 2D hydrodynamic simulations of self-gravitating discs that even brown dwarfs may be unable to maintain a gap in the disc, but instead quickly migrated inwards. Although this effect is exacerbated by the large masses of the brown dwarfs considered there it is again worth noting that the dynamical times at 100 au is long, and gap formation can take tens of orbits (although gap destruction, which is more critical here, can be much shorter). This latter effect is compensated for in my models by long outburst durations of $200 \mathrm{yr}$, compared to the $\sim 10-100$ yr usually estimated.

The very manner in which migration is implemented here implicitly assumes that the torque formulae established by Tanaka et al. (2002) and Bate et al. (2003) can be extended to planets in discs as massive as those considered here where self-gravity can dominate. While they allow useful order-of-magnitude estimates of migration rates, even these may be a long way off. If I use the Type I migration rates given by Paardekooper et al. (2010) instead of those from Tanaka et al. (2002), the migration rate of all planets drops to nearly nothing when in that regime. This is troublesome as these are specifically calculated to take into account optically thick physics in the horseshoe region around the planet, particularly important in the massive discs I consider.

This work also does not consider the time required for gap opening and closing at the end and start of an outburst, respectively. Instead, I have assumed that gap formation is instantaneous. In fact, it can take some time for a planet to carve a gap, and so the planet may remain in the Type I migration regime for far longer than the outburst duration, especially when the planet is in the outer disc when the planet's orbital period is far longer than the outburst duration. It is also unclear how much time is required for the thickened disc to fill in the gap during an outburst - if the gap- closing timescale is too long then the planet may never enter the Type I regime at all. In Appendix A, I show that the gap-closing time-scale occurs on approximately dynamical time-scales, and that conversely gap opening occurs on time-scales of tens of orbits. In this case, a planet at $\sim 100$ au might expect to remain in the Type I regime until it migrates to far smaller radii in the disc, greatly amplifying the migration rates found in the simplified models presented here. The gap is also not fully closed in these test simulations, so Type III migration may become more important, but this is sensitive to the parameters of the simulation so I defer exploring this to a future work.

I have not addressed the effect of changing the scale and mass of the disc in these models. As noted, observations of FUors by Liu et al. (2016) show that these objects are typically many hundreds to thousands of au in size. The planets I am focusing on are giants formed at large radii. However, there also exists a class of shorter and less-extreme outburst, thought to occur later in the lifetime of the system and therefore when the disc is more evolved, smaller, and less massive. These may have some effect on the migration of planets, but the short durations ( $\sim$ a year years) mean they are unlikely to be as impactful as the earlier FUor-type outbursts. Even so it is worth considering how a smaller disc may deal with such an outburst. In terms of my implementation here, it is probable that my neglect of self-consistency in modelling the outburst itself will become significant nearer to 1 au. Otherwise, the flaring nature of the disc means that $H / R$ is smaller at low $R$ so it may be harder to drive the discs out of the gap-opening regime. The effect of changing the disc mass will have an effect on the torque strengths, but using the parametrizations I have employed this will only alter the Type I migration rate (comparing equations 16 and 17, only the former is a function of the surface density $\Sigma$ ).

Of these omissions, most can be tested by running a full 3D radiation hydrodynamic simulation of the same situation, incorporating a self-consistent outburst model after the manner of Stamatellos et al. (2011) with migration torques naturally arising from the interaction between disc and planet. This is beyond the scope of this initial work so I defer it to a future paper. One omission that will be harder to test is the possibility of these planets entering the extremely rapid Type III regime (e.g. Masset \& Papaloizou 2003; Pepliński, Artymowicz \& Mellema 2008a,b,c). In this case, the rate and even direction of migration is very sensitive to the flow within the Hill radius (Pepliński et al. 2008c), which will be hard to simulate with sufficient resolution on the required time-scales. Type III requires partial gap opening and therefore could be very important as the planet transitions out of and back into the gap-opening regimes at the start and end of outbursts. It is therefore necessary to test but will likely require non-global simulations. This will also provide an excellent opportunity to test the migration rates predicted by Paardekooper et al. (2010) in this extreme regime. 


\section{CONCLUSIONS}

I have performed semi-analytic calculations of migration rates for a planet in a young circumstellar disc undergoing repeated FUortype outbursts, coupling a 1D viscous disc code to known planet migration rates. I find that planets that are normally able to maintain a gap in the disc and migrate at slow Type II rates are unable to do so during an outburst and therefore undergo rapid Type I migration for a short period. In a single outburst, I find that a $10 M_{\text {Jup }}$ planet starting at 100 au can migrate by up to 4 au in a single outburst. This allows massive planets formed early on and far out in the disc, probably by gravitational fragmentation, to migrate in rapidly under the influence of these accretion outbursts. This may in turn help to explain some fraction of observed Hot Jupiters.

\section{ACKNOWLEDGEMENTS}

I thank the anonymous referee for their thoughtful comments that helped improve the manuscript. I acknowledge support from the Science and Technology Facilities Council (STFC; grant reference ST/M000877/1).

\section{REFERENCES}

Alexander R. D., Armitage P. J., 2009, ApJ, 704, 989

Armitage P. J., 2010, Astrophysics of Planet Formation. Cambridge Univ. Press, Cambridge

Balsara D. S., 1995, J. Comput. Phys., 121, 357

Bate M. R., Lubow S. H., Ogilvie G. I., Miller K. A., 2003, MNRAS, 341, 213

Bell K. R., Lin D. N. C., 1994, ApJ, 427, 987

Bell K. R., Cassen P. M., Klahr H. H., Henning T., 1997, ApJ, 486, 372

Bertin G., Lodato G., 1999, A\&A, 350, 694

Boss A. P., 1998, ApJ, 503, 923

Cannizzo J. K., 1993, ApJ, 419, 318

Duffell P. C., MacFadyen A. I., 2012, ApJ, 755, 7

Duffell P. C., MacFadyen A. I., 2013, ApJ, 769, 41

Durisen R. H., Boss A. P., Mayer L., Nelson A. F., Quinn T., Rice W. K. M., 2007, in Reipurth B., Jewitt D., Keil K., eds, Protostars and Planets V. Univ. Arizona Press, Tucson, AZ, p. 607

Forgan D., Rice K., Cossins P., Lodato G., 2011, MNRAS, 410, 994

Hartmann L., 2009, Accretion Processes in Star Formation, 2nd edn. Cambridge Univ. Press, Cambridge

Helled R. et al., 2014, in Beuther H., Klessen R. S., Dullemond C. P., Henning T., eds, Protostars and Planets VI. Univ. Arizona Press, Tucson, p. 643

Hueso R., Guillot T., 2005, A\&A, 442, 703

Kratter K. M., Lodato G., 2016, ARA\&A, 54, 271

Lin D. N. C., Papaloizou J., 1986, ApJ, 307, 395

Liu H. B. et al., 2016, Sci. Adv., 2, e1500875

Lodato G., 2007, Riv. Nuovo Cimento, 30, 293

Lodato G., 2008, New Astron. Rev., 52, 21

Malik M., Meru F., Mayer L., Meyer M., 2015, ApJ, 802, 56

Masset F. S., Papaloizou J. C. B., 2003, ApJ, 588, 494

Mineshige S., 1986, PASJ, 38, 831

Morris J. P., Monaghan J. J., 1997, J. Comput. Phys., 136, 41

Paardekooper S.-J., Baruteau C., Crida A., Kley W., 2010, MNRAS, 401, 1950

Pepliński A., Artymowicz P., Mellema G., 2008a, MNRAS, 386, 164

Pepliński A., Artymowicz P., Mellema G., 2008b, MNRAS, 386, 179

Pepliński A., Artymowicz P., Mellema G., 2008c, MNRAS, 387, 1063

Pollack J. B., Hubickyj O., Bodenheimer P., Lissauer J. J., Podolak M., Greenzweig Y., 1996, Icarus, 124, 62

Pringle J. E., 1981, ARA\&A, 19, 137

Rafikov R. R., 2002, ApJ, 572, 566
Rice W. K. M., Armitage P. J., Wood K., Lodato G., 2006, MNRAS, 373, 1619

Rice K., Lopez E., Forgan D., Biller B., 2015, MNRAS, 454, 1940

Ruden S. P., Pollack J. B., 1991, ApJ, 375, 740

Shakura N. I., Sunyaev R. A., 1973, A\&A, 24, 337

Springel V., 2005, MNRAS, 364, 1105

Stamatellos D., Whitworth A. P., Hubber D. A., 2011, ApJ, 730, 32

Tanaka H., Takeuchi T., Ward W. R., 2002, ApJ, 565, 1257

Toomre A., 1964, ApJ, 139, 1217

Wang X.-M., 2015, MNRAS, 449, 1084

Zhu Z., Hartmann L., Calvet N., Hernandez J., Muzerolle J., Tannirkulam A.-K., 2007, ApJ, 669, 483

Zhu Z., Hartmann L., Calvet N., Hernandez J., Tannirkulam A.-K., D’Alessio P., 2008, ApJ, 684, 1281

Zhu Z., Hartmann L., Gammie C., 2009a, ApJ, 694, 1045

Zhu Z., Hartmann L., Gammie C., McKinney J. C., 2009b, ApJ, 701, 620

Zhu Z., Hartmann L., Gammie C. F., Book L. G., Simon J. B., Engelhard E., 2010a, ApJ, 713, 1134

Zhu Z., Hartmann L., Gammie C., 2010b, ApJ, 713, 1143

Zhu Z., Stone J. M., Rafikov R. R., 2013, ApJ, 768, 143

\section{APPENDIX A: TIME-SCALES FOR GAP OPENING AND CLOSING}

As noted in Section 5.2 the simple models presented in this paper do not consider the time taken for gap opening and closing. To test how these time-scales relate to the planet's orbital period I perform two simple 3D SPH simulations of an embedded planet. The first considers a planet in an unperturbed, cool disc with aspect ratio $H / R=0.05$ and demonstrates the gap-opening time-scale expected at the end of an outburst as the disc settles. The second simulation takes the output of the first after 20 orbits when a gap has been opened and raises the temperature such that the disc immediately thickens such that $H / R=0.15$, testing how long it takes for the gap to fill.

These simulations use a modified version of the public code GADGET-2 (Springel 2005), with a time-dependent Morris \& Monaghan (1997) artificial viscosity augmented by the Balsara (1995) switch. The planet is kept on a fixed orbit and the disc temperature is set by the imposed aspect ratios described above. The planet and star are simple sink particles with a sink radius $r_{\mathrm{sink}}=0.025 a_{\mathrm{p}}$, where $a_{\mathrm{p}}$ is the planet's semimajor axis. The time unit $t_{\mathrm{p}}$ is the planet's orbital period. The initial conditions contain $1.25 \times 10^{6}$ $\mathrm{SPH}$ particles. The planet mass is $5 M_{\mathrm{Jup}}$ and is kept on a fixed circular orbit.

Fig. A1 shows surface density maps of the initial conditions (left-hand panel) and the state of the simulation after 10 and 20 orbits. It is clear that the gap is still developing after 10 orbits, showing that the time-scale for gap formation is on the order of 10 orbits or longer. Conversely, Fig. A2 shows the evolution of the disc after the higher temperature has been imposed when restarting the simulation at $t=20 t_{\mathrm{p}}$. The disc is shown at $t=21,22$, and $40 t_{\mathrm{p}}$. The gap immediately fills (though not completely) within a single orbital period and remains stable for tens of orbits.

In Fig. A3, I plot $\Sigma_{\mathrm{p}} / \Sigma_{0}$ as a function of time for the gap-opening and gap-closing simulations. $\Sigma_{\mathrm{p}}$ is the surface density at the radius of the planet. $\Sigma_{0}$ is defined in the former case as the surface density at the start of the simulation, and in the latter case the surface density at the end of the simulations. Note that the two $\Sigma_{0}$ s are not the same $-\Sigma_{0}$ for the gap-closing simulation is approximately half of the value of $\Sigma_{0}$ for the gap opening, but this will vary with both disc viscosity and planet mass. 


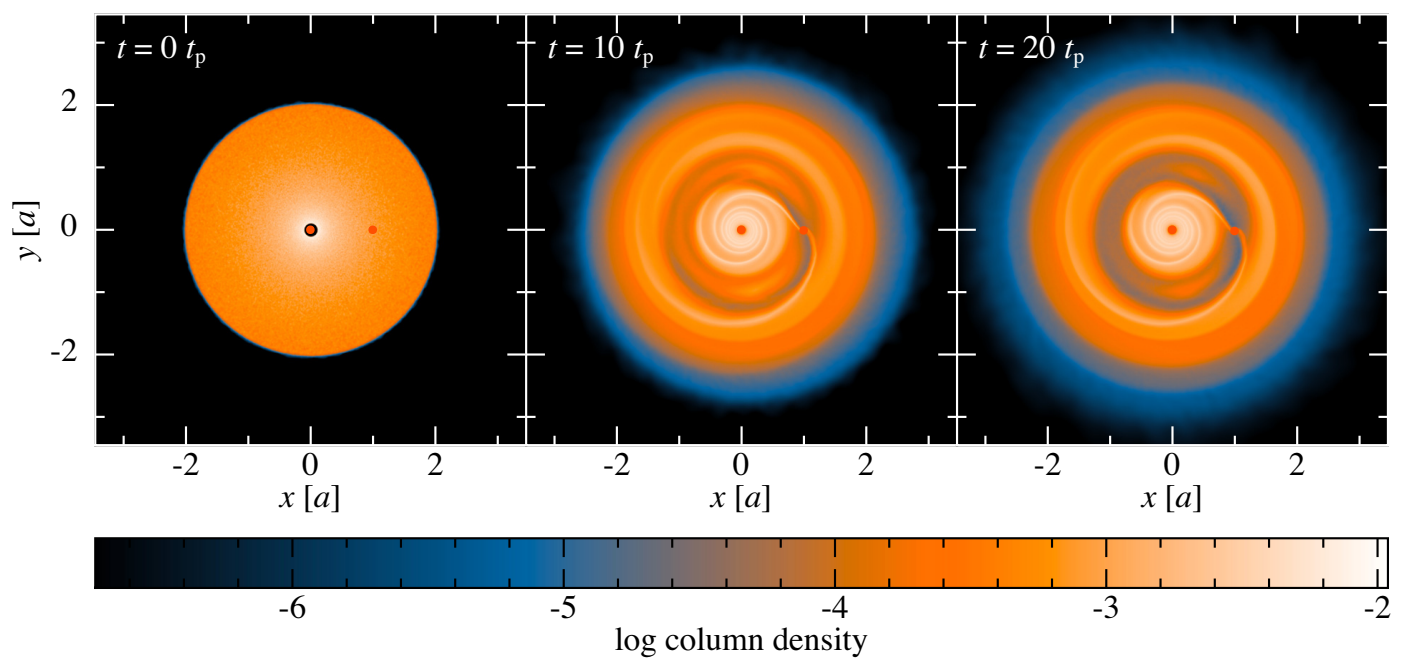

Figure A1. Surface density maps for the gap-opening simulation, after 0 (left-hand panel), 10 (middle), and 20 (right) orbits $\left(t=0 t_{\mathrm{p}}, t=10 t_{\mathrm{p}}\right.$, and $t=20 t_{\mathrm{p}}$ from left to right), showing that the gap-opening time-scale is on the order of tens of orbital periods. The surface density units are in arbitrary code units.

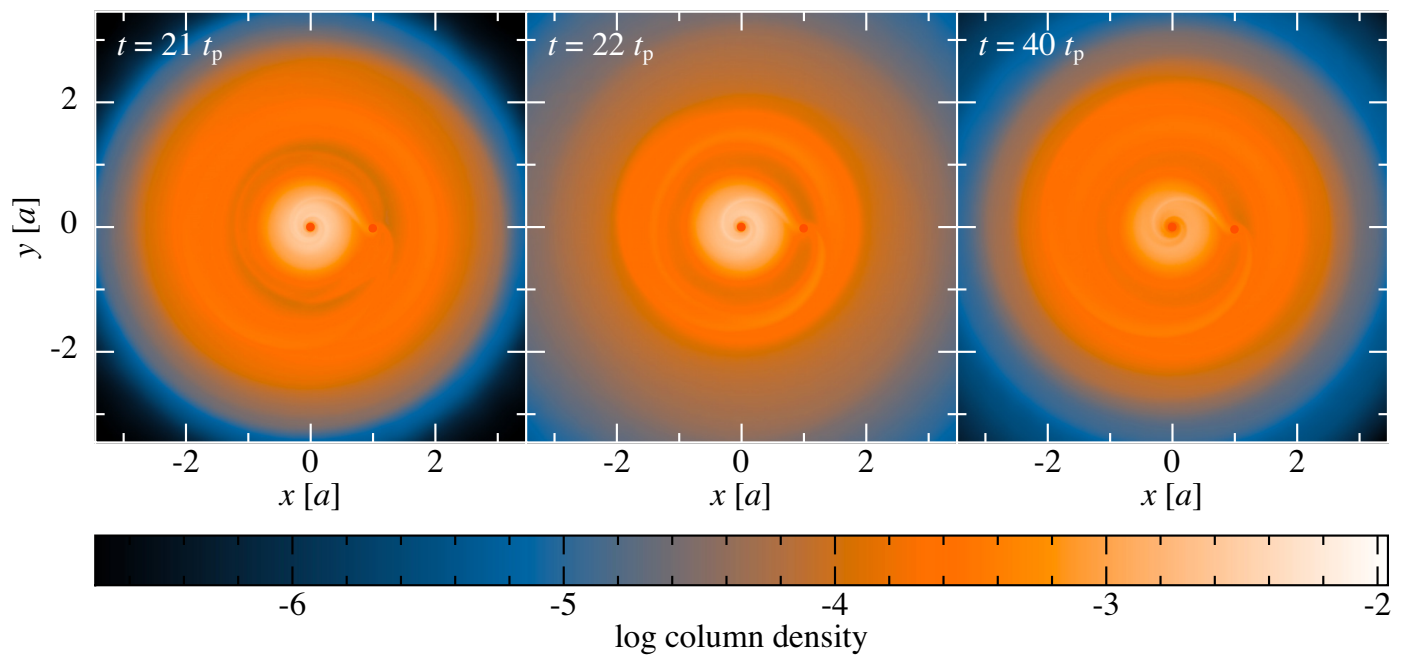

Figure A2. As Fig. A1 but for the gap-closing simulation, where the imposed disc temperature profile makes the disc thicken by a factor of three. The disc is shown 1 (left-hand panel), 2 (middle), and 20 (right) orbits after increasing the temperature and restarting the simulation $\left(t=21 t_{\mathrm{p}}, t=22 t_{\mathrm{p}}\right.$, and $t=40 t_{\mathrm{p}}$ from left to right). Compared with the gap opening shown in Fig. A1, this shows that the gap-closing time-scale is very short.

While these two simulations are far too simple to draw strong conclusions about the extent and profile of the gap formation, it clearly demonstrates that the assumption that gap closing is short is valid, provided that in more realistic conditions the same gapopening criteria dominate as in these short simulations (i.e. gap opening here is thermal and thus occurs dynamically, rather than viscous that would take orders of magnitude longer). The long time-scale for gap opening implies that the results presented in the body of this paper represent the minimal case for migration as the gap should remain closed for far longer than the outburst duration.

Fig. A4 plots the surface density profile in the gap for the gapclosing simulation (Fig. A2) showing its evolution immediately after the temperature profile of the disc is raised $\left(t=20 t_{\mathrm{p}}\right)$. If one imposes a physical radius of 100 au on the planet's semimajor axis in this test, then the outburst would end at $t=20.2 t_{\mathrm{p}}-200 \mathrm{yr}$ into the planet's $1000 \mathrm{yr}$ orbit. Even though this is just a fraction of the orbital time, the gap profile has significantly changed, indicating that although the gap may not fully close in this short interval the planet's circumstances have changed enough that it will likely enter a different migration regime until the gap opens again. Interestingly, as the gap may only be partial, the planet may enter the poorly understood Type III migration regime, which is typically much faster than Type I migration. A number of caveats must be borne in mind here - particularly that the gap-opening/closing process is a strong function not only of temperature and planet mass but also of disc viscosity and thermodynamic treatment. Therefore, due to the simplicity of the models here I leave further investigation of this point to future work using full $3 \mathrm{D}$ radiation hydrodynamics. 


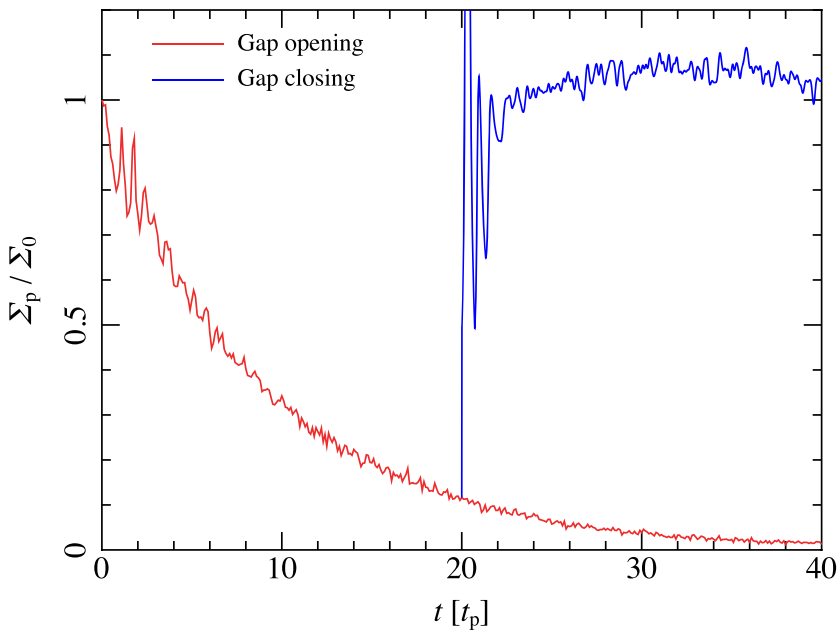

Figure A3. Evolution of the disc surface density at the planet's radius $\Sigma_{\mathrm{p}}$, tracking the gap depth. $\Sigma_{0}$ is the value of $\Sigma_{\mathrm{p}}$ at the start of the simulation in the case of the gap-opening run, and the value at the end of the simulation for the gap-closing run. Note that the two values of $\Sigma_{0}$ are not the same. This figure shows that the gap-closing time-scale is much shorter than the gap-opening time-scale, with the former being less than one orbital period.

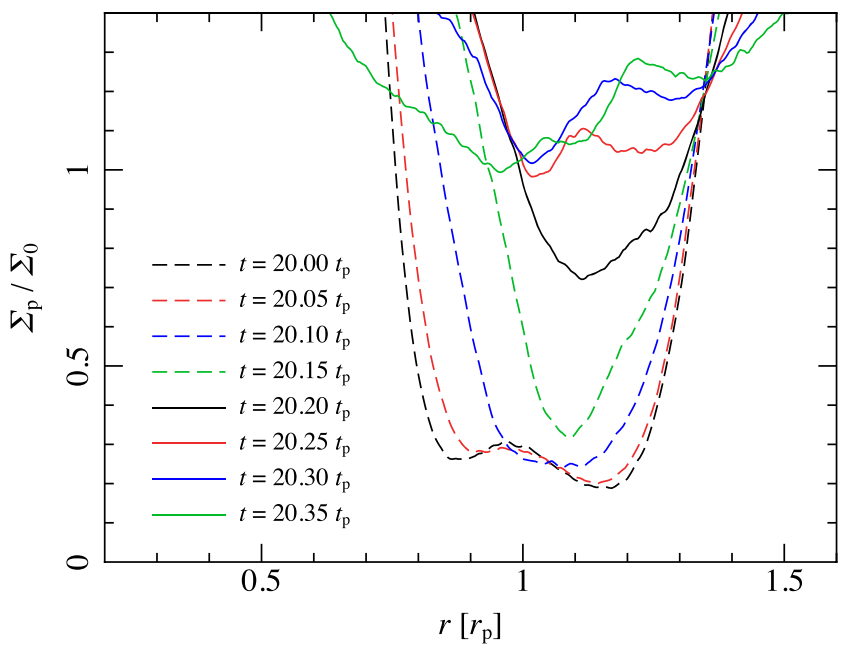

Figure A4. Evolution of the disc surface density profile at the planet's radius $\Sigma_{\mathrm{p}}$ for the gap-closing simulation, showing the evolution of the gap profile immediately after the temperature of the disc is raised. $\Sigma_{0}$ here is as in Fig. A3. It is clear that although the gap is still in the process of closing by $t=20.2 t_{\mathrm{p}}$ (solid black line) the gap is still significantly shallower at this point and the planet's migration behaviour must change.

This paper has been typeset from a $\mathrm{T}_{\mathrm{E}} \mathrm{X} / \mathrm{L} \mathrm{T}_{\mathrm{E}} \mathrm{X}$ file prepared by the author. 